\title{
Psychological Resilience as an Emergent Characteristic for Well-Being: A Pragmatic View
}

\author{
Peter Kay Chai Tay ${ }^{a, b}$ Ka Keat Limc ${ }^{c, d}$ \\ ${ }^{a}$ Health and Social Sciences, Singapore Institute of Technology, Singapore, Singapore; ${ }^{b}$ Centre for \\ Ageing Research and Education, Duke-NUS, Singapore, Singapore; ' Health Services and Systems Research, \\ Duke-NUS, Singapore, Singapore; ${ }^{d}$ Department of Population Health Sciences, Faculty of Life Sciences \& \\ Medicine, King's College London, London, UK
}

\section{Keywords}

Pragmatism · Successful aging · Biopsychosocial ·

Psychological resilience $\cdot$ Well-being

\begin{abstract}
Currently, there is no clear agreement on the definition and conceptualization for psychological resilience (PR) in old age. Adopting a pragmatic view, this article elucidates the definition and conceptualization of PR by (1) extracting existing PR concepts that are relevant to aging, and (2) elucidating the contributing and delimiting factors for developing and sustaining PR as guided by the biopsychosocial framework. In addition, a comprehensive review of the tools used to measure PR was conducted to examine how scholars have conceptualized PR. Consequently, the renewed understanding defines PR as an emergent construct which is malleable, enduring, and can be developed and sustained by a dynamic interplay of biological, psychological, spiritual, and social factors. Correlates in terms of physical and mental well-being in the context of aging are identified.
\end{abstract}

(c) 2020 S. Karger AG, Basel

$\begin{aligned} & \text { karger@karger.com } \\ & \text { www.karger.com/ger }\end{aligned}$
Karger 2020 S. Karger AG, Basel

\section{Introduction}

Psychological resilience (PR) has been a topic of research since the 1980s (online suppl. ref. 7; see www. karger.com/doi/10.1159/000509210 for all online suppl. material). Currently, the definitions and conceptualizations of PR remain nebulous, particularly for practitioners, policymakers, and individuals who want to understand PR and enhance it. While previous reviews on PR clarified the general definition of PR or focused on psychological interventions which might have a limited application in policy-making at the population level (online suppl. ref. 7), this article uses a pragmatic approach, focusing on older adults from an individual level to population level.

A pragmatic approach is an approach that defines, conceptualizes, and identifies factors in ways that could contribute to the development and maintenance of PR. In addition, it focuses on the needs of older people and person-centered care. We aimed to achieve this by elucidating PR as a developmental concept for older adults based on the biopsychosocial model. This model has been used to understand needs among older adults holistically, en- 
abling this review to extend beyond current conceptualizations of PR by, for instance, including spiritual factors (online suppl. ref. 7). We proffer that PR is malleable, and thus can be trained, enhanced, and maintained. This renewed understanding is used to examine how PR can affect the physical and mental well-being of older people. We adopted this pragmatic approach because (i) currently, PR has numerous definitions and conceptualizations, and is therefore difficult to measure, and the existing measures may not capture PR holistically; and (ii) understanding how PR affects physical and mental well-being downstream will be useful for developing more targeted interventions that can then be applied to the specific mechanisms that drive PR. This is especially relevant for older adults because they tend to encounter more numerous physical and psychosocial challenges (e.g., the death of a spouse) than younger people.

Historically, PR research centered on at-risk children and adolescents who overcame adversity due to a challenging background (e.g., economic hardship), stressful circumstances (e.g., racial discrimination) as well as political violence and wars $[1,2]$. It was argued that PR in older people was understudied [3]. PR is especially relevant in old age because older people tend to encounter more stressful life events than younger people, like health issues, caring for a spouse, and bereavement [4]. In addition, PR in some older adults is qualitatively different from in younger adults due to different life perspectives and experiences in matters related to romance, family, work, death, and dying [3]. Such differences are highlighted by the socioemotional selectivity theory which states that, in older age, time is perceived as limited, so close relationships and emotion regulation are prioritized over other life goals like accumulating resources and attracting a mate which are important at a younger age [5]. Given these distinctions between older and younger people, the development of PR as well as the factors which contribute to PR during old age should be considered in the light of the experiences and perceptions that are specific to older people.

\section{What Is Psychological Resilience?}

\section{Definitions}

The Oxford dictionary defines resilience as the ability to "rebound or spring back" [6]. A review article revealed 5 key concepts related to PR including "rising above to overcome adversity," "adaptation and adjustment," "ordinary magic," "good mental health," and "ability to

Psychological Resilience and Well-Being bounce back" [7]. Other scholars provide more specific definitions such as "effective coping and adaptation although faced with loss, hardship, or adversity" [8], or extend the definition to include "flourishing despite adversity," which has been highlighted in the literature on successful aging [9]. Flourishing is a significant concept given that it refers to well-being beyond recovery and returning to baseline psychological state and includes achieving human excellence through deep engagement in life purposes and rich experiences in interpersonal relationships [10]. Defining PR as distinct from physical resilience (health) is relevant because many older adults will develop health problems [11], yet the mental well-being can remain unaffected by one's health condition [12].

The importance of PR in old age is exemplified by the notion of salutogenesis, where health is more holistically defined based on the biopsychosocial view and the understanding that psychosocial factors contribute to physical health [13]. Apart from having biological bases [e.g., 14], $\mathrm{PR}$ is also dynamic in its emergence and activation. Understanding this is important because it suggests that a person's PR can be developed and has to be constantly nourished. Taken together, PR is currently defined as a dynamic ability, developed and supported by biopsychosocial and spiritual factors, that enables an older person to spring back and flourish in the face of adversity.

\section{Conceptualizations in the Literature}

PR has been conceptualized as a trait, state, trajectory, response function, outcome, or a mix of these concepts. PR conceptualized as a person-level trait suggests that it is largely driven by predisposition factors and is somewhat unchangeable [15-17]. This position is consistent with the notion that biological factors such as hormones influence psychological factors [18]. In contrast, state, trajectory, response function, and outcome suggest that PR is developed or subjected to situational fluctuations and social and environmental influences (online suppl. ref. 5). Given that community- and family-level factors such as the presence of close confidant relationships have also been postulated as components that contribute to PR [16], other scholars such as Flach [19] adopt a more flexible, state-based view of PR as a process which can be learnt at any point throughout life. Process PR has also been conceptualized as an iterative developmental process between disruption (i.e., stressful life encounters) and reintegration (i.e., resolution) [19]. Related to this, Richardson [17] (online suppl. ref. 7) described that PR developed when exposure to stressful situations, leading to an inoculating effect, enables one to cope better in fu- 
Fig. 1. Biological (Bio), psychological (Psycho), social (Soc), and spiritual (Spiritual) factors interact in the development and maintenance of psychological resilience (PR) among older adults. Consequently, PR buffers the impact of adversities, leading to better physical and mental well-being outcomes, and vice versa.

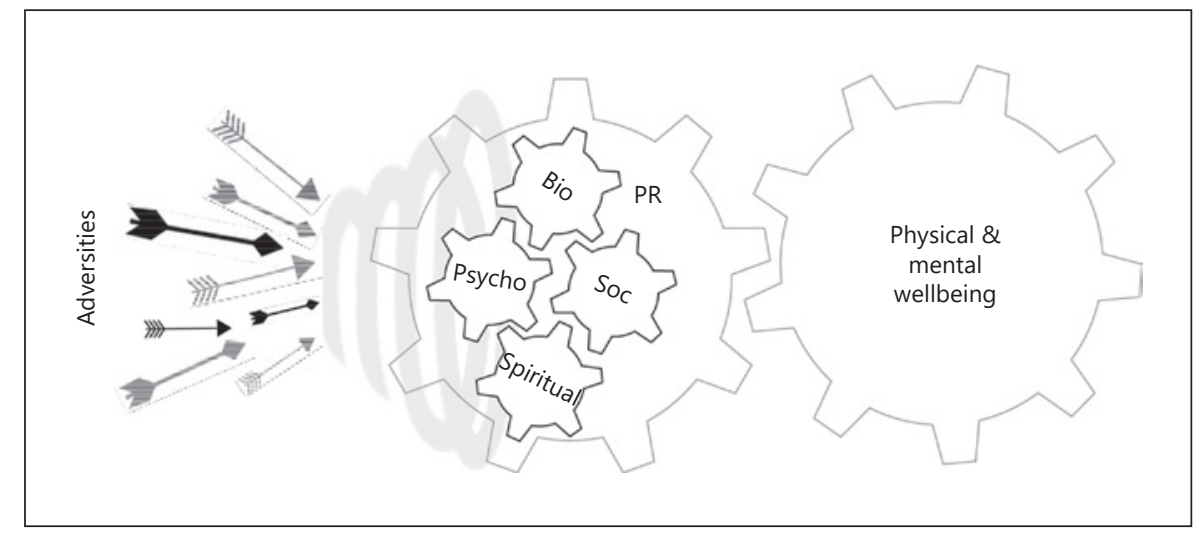

ture encounters; this is also termed adversarial growth, i.e., response function. In sum, we focus here on the statebased features, to facilitate a pragmatic approach to understanding PR as a developmental concept.

\section{Measurements}

Apart from examining the extant definitions of PR, reviewing the tools used to measure it provides another way for us to understand how scholars conceptualize it. We identified 15 tools developed to measure PR to date. The online supplementary material shows the concepts incorporated for each tool and its correlates, followed by an analytical summary of all the tools.

In the following section, we conceptualize PR in old age, based on a pragmatic approach by (i) extracting features of PR identified from the literature which are relevant to older people in terms of developing and maintaining $\mathrm{PR}$, and (ii) using the biopsychosocial model to explain the contributing and delimiting factors for PR. We then examine how these factors are related to physical and mental well-being in older adults.

\section{Conceptualizing PR for Older Adults}

The biopsychosocial model highlights the dynamic interplay involving biological, psychological, and social factors, and serves as a theoretical guide in providing a holistic and organized account of a health condition or psychological construct [e.g., 12, 20]. Given the multitude of contributing and delimiting factors associated with PR, using the biopsychosocial model to elucidate PR as the outcome is specifically relevant for older adults.

Apart from using the biopsychosocial framework to elucidate factors underlying the emergence of PR, our pragmatic approach includes extracting concepts that are relevant to the development and maintenance of PR (e.g., adopting characteristic rather than trait PR). Conceptualizing PR as a characteristic specifically suggests that it can change over time or through intervention, as opposed to a person-level trait which is largely fixed [15]. In addition, a characteristic is distinguishable from a state, to the extent that characteristic PR is developed through cumulative experience and learning whereas state pertains to more contextual and transient aspects that tend to vary across time and situations. In contrast to trait, state, response function, and outcome, characteristic PR is malleable, enduring, and can be influenced by a multitude of factors. PR therefore requires sustained maintenance efforts. While intraindividual variations in $\mathrm{PR}$ have been observed [21, 22], PR can nevertheless be enduring for an individual once it is sufficiently developed and consistently maintained [23]. Based on this renewed understanding, we outline the factors associated with the emergence and maintenance of PR specific to older adults, and the implications for their physical and mental well-being (Fig. 1). Using the Loads-Levers-Lifts analogy, Hildon et al. [20] proposed that loads in one biopsychosocial domain (e.g., diabetes) can affect other domains (e.g., depression) and general physical and mental health. Levers refer to interventions and policies (e.g., occupational therapy) that support the lifts across the domains (e.g., self-efficacy). In this review, the emergent PR is conceptualized as the interactional outcome of the lifts and loads (i.e., the contributing and delimiting factors, respectively) across the domains.

\section{Contributing and Delimiting Factors}

In this section, we delineate the contributing and delimiting factors related to the development and maintenance of PR which moderates the relationship between the effects of adversities and well-being outcomes. Effects 
of adversities include physical and mental stressors such as functional disabilities and bereavement. Well-being outcomes in the form of positive physical and mental well-being are discussed in the following section.

\section{Biological Factors}

PR may be implicated in basic biological systems that have genetic bases and are neurobiologically expressed. Biological studies have demonstrated that PR is subjected to the effects of challenges to the stability and homeostasis of a physiological system [e.g., 24]. Despite the link found between biological systems and PR, understanding the effect of modifiable factors such as overt behaviors and perceptions is more pragmatic in the context of aging. The literature relating to the biopsychosocial perspective of health and well-being emphasizes how physical factors affect human cognition and psychological state [20]. For instance, the socioecological model for PR states that objective health status such as poor health and limitations in instrumental ADLs can adversely affect PR [25]. Similarly, subjective health status, as indicated by self-rated health and energy levels, is also positively correlated with PR [3] (but see also [26]). Furthermore, physical fitness is another pathway towards stimulating PR [14]. Older adults who engage in physical activities and aerobic exercises report higher indicators of PR [27]. Conversely, Resnick [28] argued that PR can also enable one to overcome the physical challenges and barriers to engage in exercise, suggesting that PR and physical activities may be mutually reinforced, and that more work to examine the relationship between the two could be a fruitful avenue towards simultaneously building better psychological and biological health. Thus, engaging in physical activities contributes towards PR which may, in turn, moderate the negative impact of adversity on physical and mental well-being.

\section{Psychological and Spiritual Factors}

Psychological factors include emotional and cognitive factors. Spiritual factors include religiosity and spirituality. Religiosity refers to the presence of religious belief and the level of involvement in religious activities (e.g., attending church services) whereas spirituality is defined as the search for meaning in life and a view of the world as larger than oneself [29]. A wealth of research has revealed that positive emotions and adaptive cognition moderate the effects of negative emotions when people encounter stressful life events $[8,21,30]$, leading to desirable well-being outcomes such as greater life satisfaction [31]. Notably, resilient older adults tend to use emotion-

Psychological Resilience and Well-Being al regulation and problem-solving abilities more than younger adults [3]. For instance, positive emotions moderate the negative effects of daily stress and mental wellbeing among elderly widows by facilitating effective emotional recovery from the stress of bereavement [23]. On the other hand, PR is associated with adaptive cognition like problem-solving and learning from experience more than less adaptive cognition e.g., an avoidant style of coping [9]. Generativity, a concept similar to active aging and productive aging, promotes adaptive cognition, which potentially leads to flourishing in old age. Specifically, agentic generativity is achieved through strengthening the self by engaging in creative pursuits as well as productive and leadership activities [32]. These qualities foster the adaptive cognition component of PR by developing abilities such as problem-solving and selfawareness [33].

Spiritual factors can enhance and support PR among older adults. Spirituality represents an integral part of the resiliency model, where Richardson [17] ascribed biopsychospiritual homeostasis to one's adaptation to stressful situations. This is specifically relevant to those older adults who draw strength from their spiritual orientation, whether in the form of God, chi, quanta, or spirit [17]. Spirituality per se moderates the effects of stressful life events and depression [29]. Notably, longitudinal studies lending support to the causal effect of emotional and cognitive factors suggest that promoting positive emotions and adaptive cognitive coping styles can moderate the negative effects of adversity on mental well-being among older adults [21, 23]. Nonetheless, the causal effects of engaging spiritual beliefs and practices remain to be further explored in future longitudinal research.

\section{Social Factors}

Having strong social networks and support builds resources in the form of social capital [34], enabling older adults to overcome adversities [35]. Specifically, a social network contributes to the development and maintenance of PR [36], and is important during, as opposed to after and in response to, the adversity [35]. Most older adults focus on a few relationships which they perceive as secure and emotionally satisfying (i.e., Socioemotional Selectivity Theory, 5). Nonetheless, beneficial social networks can extend beyond one's family and friends. PR can be developed through networks across all layers of society, from the familial to the community level [36]. For instance, social support through engaging in community activities was associated with greater PR in a group of older men living with HIV [37]. 
Given the rising prevalence of loneliness and isolation in the elderly, participating in community activities may be an effective alternative for building social networks among older adults who live alone. For older adults, social networks can be strengthened as understood through the notion of generativity. Developing communal generativity by participating in social activities such as mentoring the younger generation, caring for grandchildren, or volunteering at social care centers can maintain social networks and enrich older people's social lives [32]. PR could be enhanced by such social contributions since older people can draw on this social capital when they subsequently encounter physical or mental adversity.

The socioecological model of PR identified social risk factors including a low level of education, financial strain, and being unmarried as factors that affect PR [25]. In contrast, stressful life events which occur earlier in life, such as having been in a war and/or experiencing terrorist attacks, may have inoculating consequences for older people, especially when the adversities were successfully conquered. For instance, older male war veterans who had experienced combat exposure were found to cope better with the emotional strain of care-giving [38]. Governments and social institutions are in the position of promoting PR by providing the necessary ecological infrastructures and social resources. For instance, developing community programs that encourage civic engagement in the form of volunteering may be an avenue to promote and sustain PR.

\section{Implications for Physical and Mental Well-Being}

In this section, we explain how PR can moderate the association between physical and mental stressors in old age, and physical and mental well-being outcomes. PR has been observed to moderate the impact that functional disabilities and various forms of illnesses have on wellbeing. For instance, centenarians with greater PR tend to have better self-rated health, and lower mortality, cognitive impairment, and Activities of Daily Living (ADLs) disabilities [39]. As many older adults suffer from multiple chronic conditions, PR serves a critical function against further physical decline. Specifically, PR mitigates the effect of chronic conditions on a subsequent decline in ADLs and instrumental ADLs (online suppl. ref. 11), while increasing the likelihood that older adults with multiple chronic conditions will remain in the workforce [25]. Thus, older adults with greater PR not only overcome the challenges associated with functional disability and health problems but are also less likely to experience further decline in physical functioning.

PR moderates the negative effects of adversity on mental well-being in contexts relating to bereavement, depression, anxiety, and the psychological problems induced by chronic conditions [22, 37]. Compared to younger people, older adults are more likely to encounter the loss of a loved one including a spouse, family members, or friends, and dealing with the loss of a loved one is an extremely distressing experience. Nonetheless, nearly half of older adults are able to harness PR to manage their emotional upheaval [22]. Cognitive mechanisms and social connectedness, in particular, may account for the better bereavement outcome. Among a group of older widowed adults, a sense of control and social support was experienced by those identified as resilient based on their grief scores, and the researchers suggest that the widow/ers deployed social support for maintaining psychoemotional stability and exercised personal control to manage the changes after their bereavement (online suppl. ref. 11). Besides viewing PR within a deficit model, it has also been proffered to enhance mental well-being in the context of successful aging and life-long learning despite age-related illnesses, disabilities, and unfavorable social conditions. For instance, resilient older workers tend to remain employed, because PR can mitigate susceptibility towards negative perceptions and stereotyping, and it enables these individuals to find meaning in their work and participate in meaningful activities such as volunteering [40].

\section{Discussion}

In this review, we have used a pragmatic approach to conceptualize PR for older adults and elucidate the factors which contribute to the development and maintenance of PR among older adults. We conceptualize PR in old age as an emergent characteristic that is malleable and enduring, and represents a complex, integrative construct with a multitude of biopsychosocial and spiritual contributing factors. These factors correspond to the "loads" and "lifts," as already mentioned in the Loads-Lever-Lifts analogy, interacting and affecting the development and sustainability of PR. For instance, the sympathetic nervous system and hypothalamic-pituitary-adrenocortical system are concurrently dependent on social attachment and are involved in stress management [41]. Conversely, positive emotions deployed as PR mitigates physiological arousal during times of distress lead to an improved con- 
nection between the mind and body [8]. Consequently, PR mitigates physical and mental deterioration but also has the potential to enhance physical and mental wellbeing in old age.

PR has been conceptualized in other ways such as posttraumatic growth, which can be described as a process leading to the development of PR. Other related constructs include grit, self-efficacy, personal mastery, and stress-related growth. In addition, some scholars argue that $\mathrm{PR}$ is essentially a response function. In other words, $\mathrm{PR}$ is not a construct as described here, but an observed outcome when a person's biopsychosocial functioning demonstrates a "bounce back" following adversity [e.g., 42]. For instance, a bounce back can be observed where a person's mood returns to baseline after a period of depression after the death of their spouse. How quickly and smoothly the person's mood returns to baseline can be described as PR as well. PR is therefore described as a trajectory of improving physical and mental functioning over time, or the variance across individuals, as opposed to how we conceptualized PR as a construct in and of itself. The notion of PR as a trajectory thus renders the development of a "gold standard" measure and a definition of PR less probable. We thus preferred the current conceptualization of PR as an ability. It is also more pragmatic, given that it is a measurable construct, than PR as a response function which requires repeated measures of well-being over an extended period of time with the factors contributing to PR development and maintenance being less clear.

\section{Further Considerations and Future Directions}

We have conceptualized PR as an emergent construct relevant to the well-being of older people. We have also argued that it is worthwhile to bolster and maintain PR in people from a younger age. Thus, programs such as cognitive behavioral therapy (CBT) and mindfulness training, based on the biopsychosocial framework of populations like retiring or late-career adults may be fruitful. Such programs represent the "levers" in the Loads-Levers-Lifts model. Mindfulness training has demonstrated effectiveness across different populations such as improved mental health in military and veteran communities [43], reduced stress, greater positive risk-taking and enhanced resilience among adolescents, and reduced distress among informal carers of individuals with intellectual disabilities [44]. Longitudinal research that evaluates the effectiveness of these programs will be informative. In addition, governments have tremendous opportunities to provide "levers" by supporting other social agencies to create environments where older adults can flourish, for instance, enabling and empowering people to work longer if they wish to, and involving older people in policymaking and other key decisions can ultimately contribute to PR [45]. In addition, tackling ageist practices is crucial in facilitating these plans $[45,46]$.

It is noteworthy that while only a few studies have examined the causal factors for PR [21,23], most studies are correlational. As such, more longitudinal research is warranted to examine whether causal factors, as outlined here, do indeed contribute to PR. Statistical methods such as Growth Mixture Modeling which enables the identification of discrete trajectories following adversity may facilitate this [47]. Bonanno et al. [48] highlighted 4 temporal elements to consider in longitudinal analyses of PR including: whether the PR is measured at baseline or preadversity functioning, contextualizing the adversity circumstances, post-adversity adjustment, and elucidating the predictors of resilient outcomes that include community and family resilience.

Several studies conceptualized PR as a change score for adversity [e.g., 14, 35, 49]. For instance, an observed improvement for bereavement such as a better grief outcome indicates $\mathrm{PR}$, but a poorer grief outcome indicates a lack of PR [35]. However, the causal factors for PR remain unknown in these studies. This paper provides a more holistic framework for future studies to measure PR in older people.

\section{Limitations}

One limitation of this mini-review has to do with the focus on within-individual factors based on the biopsychosocial model. Beyond within-individual factors, macro-factors including sociopolitical and cultural influences may affect PR. It is particularly necessary to acknowledge that there is unequal access to resources among older people in certain cultures [20]. Thus, older people without adequate access to socioeconomic resources will experience difficulties building PR. For instance, a society with no focus on inclusiveness of older people, such as taking steps to develop age-friendly environments and promoting anti-ageism, will impede the development of PR in this population [46]. We examined PR in the older population as a whole. Individual differences such as gender and ethnic differences are beyond the purview of this paper and should be explored further, based on a biopsychosocial framework. For instance, PR appears to be greater in women than in men, particularly older women [35]. Fu- 
ture research should investigate in which domains such gender differences are observed. Notwithstanding these limitations, this model shows how biopsychosocial factors are connected to PR, and it delineates the physical and mental well-being outcomes as a result of possessing PR.

\section{Conclusion}

Our conceptualization of $\mathrm{PR}$ is consistent with the notion of person-centered care, given that $\mathrm{PR}$ is elucidated from a comprehensive set of factors using a pragmatic approach. Considering that the constituents of adversity are subjective and vary across individuals, person-centered care will rely much on clinician assessment; our conceptualization enables a more holistic assessment of older people. We hope that this understanding of PR will be incorporated into work with this objective. In addition, including and evaluating programs such as CBT and mindfulness training whenever appropriate can promote better physical and mental well-being in older adults, not just in ways that would ultimately lead to "bouncing back" from adversities but also in terms of successful aging.

\section{Acknowledgements}

This review was supported by the Centre for Ageing Research and Education, Duke-NUS Medical School. We would like to thank Dr. Truls Østbye for his comments on the initial drafts of the manuscript.

\section{Conflict of Interest Statement}

The authors declare no conflicts of interest.

\section{Funding Sources}

The writing of this paper was not supported by any funding.

\section{Author Contributions}

P.K.C.T. and K.K.L. assembled the literature. P.K.C.T. wrote the initial draft of the manuscript. Both authors contributed equally to implementing and revising the manuscript.

\section{References}

1 Werner EE. Protective factors and individual resilience. In: Meisels S, Shonkoff J, editors. Handbook of Early Childhood Intervention. Cambridge: Cambridge University Press; 1990.

2 Masten AS. Resilience comes of age: Reflections on the past and outlook for the next generation of research. In: Glantz M, Johnson J, Huffman L, editors. Resilience and Development: Positive Life Adaptations. New York: Plenum; 1999. pp. 281-96.

3 Gooding PA, Hurst A, Johnson J, Tarrier N. Psychological resilience in young and older adults. Int J Geriatr Psychiatry. 2012 Mar;27(3):262-70.

4 Stroebe MS, Hansson RO, Stroebe W, Schut $\mathrm{H}$, editors. Bereavement and old age. In: Handbook of Bereavement Research. Consequences Coping, and Care. Washington (DC): American Psychological Association; 2001. pp. 241-61.

5 Carstensen LL. The influence of a sense of time on human development. Science. 2006 Jun;312(5782): 1913-5.

6 Oxford English Dictionary. Compact Edition of the Oxford English Dictionary. Volume II. Oxford: Oxford University Press; 1971.

7 Aburn G, Gott M, Hoare K. What is resilience? An integrative review of the empirical literature. J Adv Nurs. 2016 May;72(5):980-1000.

8 Tugade MM, Fredrickson BL. Resilient individuals use positive emotions to bounce back from negative emotional experiences. J Pers Soc Psychol. 2004 Feb;86(2):320-33.
9 Hildon Z, Montgomery SM, Blane D, Wiggins RD, Netuveli G. Examining resilience of quality of life in the face of health-related and psychosocial adversity at older ages: what is "right" about the way we age? Gerontologist. $2010 \mathrm{Feb} ; 50(1): 36-47$.

10 Ryff CD, Singer B. The Contours of Positive Human Health. Psychol Inq. 1998;9(1):1-28.

11 Belikov AV. Age-related diseases as vicious cycles. Ageing Res Rev. 2019 Jan;49:11-26.

12 Hildon Z, Smith G, Netuveli G, Blane D. Understanding adversity and resilience at older ages. Sociol Health Illn. 2008 Jul;30(5):72640.

13 Antonovsky A. The salutogenic model as a theory to guide health promotion 1 . Health Promot Int. 1996;11(1):11-8.

14 Silverman MN, Deuster PA. Biological mechanisms underlying the role of physical fitness in health and resilience. Interface Focus. 2014 Oct; 4(5):20140040.

15 Whitson HE, Duan-Porter W, Schmader KE, Morey MC, Cohen HJ, Colón-Emeric CS. Physical Resilience in Older Adults: Systematic Review and Development of an Emerging Construct. J Gerontol A Biol Sci Med Sci. 2016 Apr;71(4):489-95.

16 Jacelon CS. The trait and process of resilience. J Adv Nurs. 1997 Jan;25(1):123-9.

17 Richardson GE. The metatheory of resilience and resiliency. J Clin Psychol. 2002 Mar; 58(3):307-21.
18 Miller TW. Advances in understanding the impact of stressful life events on health. Hosp Community Psychiatry. 1988 Jun;39(6):61522.

19 Flach FF. Resilience: Discovering a new strength at times of stress. New York: Fawcett Columbine; 1988.

20 Hildon ZJ, Tan CS, Shiraz F, Ng WC, Deng X, Koh GC, et al. The theoretical and empirical basis of a BioPsychoSocial (BPS) risk screener for detection of older people's health related needs, planning of community programs, and targeted care interventions. BMC Geriatr. 2018 Feb;18(1):49.

21 Tugade MM, Fredrickson BL, Barrett LF. Psychological resilience and positive emotional granularity: examining the benefits of positive emotions on coping and health. J Pers. 2004 Dec;72(6):1161-90.

22 Spahni S, Morselli D, Perrig-Chiello P, Bennett KM. Patterns of Psychological Adaptation to Spousal Bereavement in Old Age. Gerontology. 2015;61(5):456-68.

23 Ong AD, Bergeman CS, Bisconti TL, Wallace KA. Psychological resilience, positive emotions, and successful adaptation to stress in later life. J Pers Soc Psychol. 2006 Oct;91(4): 730-49.

24 Hunter RG, McEwen BS. Stress and anxiety across the lifespan: structural plasticity and epigenetic regulation. Epigenomics. 2013 Apr;5(2):177-94. 
25 Jason KJ, Carr DC, Washington TR, Hilliard TS, Mingo CA. Multiple Chronic Conditions, Resilience, and Workforce Transitions in Later Life: A Socio-Ecological Model. Gerontologist. 2017 Apr;57(2):269-81.

26 Nygren B, Aléx L, Jonsén E, Gustafson Y, Norberg A, Lundman B. Resilience, sense of coherence, purpose in life and self-transcendence in relation to perceived physical and mental health among the oldest old. Aging Ment Health. 2005 Jul;9(4):354-62.

27 Talsma AN. Evaluation of a theoretical model of resilience and select predictors of resilience in a sample of community-based elderly. 1996, University of Michigan: Unpublished doctoral dissertation.

28 Resnick B. Conclusion. In: Resnick B, Gwyther LP, Roberto KA, editors. Resilience in Aging: Concepts, Research, and Outcomes. New York: Springer; 2011.

29 Maddi SR, Brow M, Khoshaba DM, Vaitkus M. Relationship of hardiness and religiousness to depression and anger. Consult Psychol J. 2006;58(3):148-61.

30 Zautra AJ, Johnson LM, Davis MC. Positive affect as a source of resilience for women in chronic pain. J Consult Clin Psychol. 2005 Apr;73(2):212-20.

31 Cohn MA, Fredrickson BL, Brown SL, Mikels JA, Conway AM. Happiness unpacked: positive emotions increase life satisfaction by building resilience. Emotion. 2009 Jun;9(3):361-8.

32 Villar F. Successful ageing and development: the contribution of generativity in older age. Ageing Soc. 2012;32(7):1087-105.

33 Focquaert F, Platek SM. Social Cognition and the Evolution of Self-Awareness. In: Platek SM, Keenan JP, Shackelford TK, editors. Evolutionary Cognitive Neuroscience. Cambridge (Massachusetts): The MIT Press; 2007. p. 457.

34 Elliott JR, Haney TJ, Sams-Abiodun P. Limits to social capital: comparing network assistance in two New Orleans neighborhoods devastated by Hurricane Katrina. Sociol Q. 2010;51(4):624-48.
35 Netuveli G, Wiggins RD, Montgomery SM, Hildon Z, Blane D. Mental health and resilience at older ages: bouncing back after adversity in the British Household Panel Survey. J Epidemiol Community Health. 2008 Nov; 62(11):987-91.

36 Sippel LM, Pietrzak RH, Charney DS, Mayes LC, Southwick SM. How does social support enhance resilience in the trauma-exposed individual? Ecol Soc. 2015;20(4):10.

37 Emlet CA, Shiu C, Kim HJ, Fredriksen-Goldsen K. Bouncing Back: Resilience and Mastery Among HIV-Positive Older Gay and Bisexual Men. Gerontologist. 2017 Feb;57 suppl 1:S40-9.

38 Monin JK, Levy BR, Pietrzak RH. From serving in the military to serving loved ones: unique experiences of older veteran caregivers. Am J Geriatr Psychiatry. 2014 Jun;22(6): 570-9.

39 Gu D, Feng Q. Psychological Resilience of Chinese Centenarians and Its Associations with Survival and Health: A Fuzzy Set Analysis. J Gerontol B Psychol Sci Soc Sci. 2018 Jun; 73(5):880-889.

40 James JB, Besen E, Pitt-Catsouphes M. Resilience in the Workplace: Job Conditions that Buffer Negative Attitudes Toward Older Workers. In: Resnick B, Gwyther LP, Roberto KA, editors. Resilience in Aging: Concepts, Research, and Outcomes. New York (NY): Springer New York; 2011. pp. 33149.

41 Loman MM, Gunnar MR; Early Experience, Stress, and Neurobehavioral Development Center. Early experience and the development of stress reactivity and regulation in children. Neurosci Biobehav Rev. 2010 May; 34(6):867-76
42 Gijzel SMW, van de Leemput IA, Scheffer M, Roppolo M, Olde Rikkert MGM, Melis RJF. Dynamical Resilience Indicators in Time Series of Self-Rated Health Correspond to Frailty Levels in Older Adults. J Gerontol A Biol Sci Med Sci. 2017 Jul;72(7):991-6.

43 Thomas KH, Taylor SP. Bulletproofing the psyche: mindfulness interventions in the training environment to improve resilience in the military and veteran communities. Adv Soc Work. 2015;16(2):312-22.

44 McConachie DA, McKenzie K, Morris PG, Walley RM. Acceptance and mindfulnessbased stress management for support staff caring for individuals with intellectual disabilities. Res Dev Disabil. 2014 Jun;35(6): 1216-27.

45 Beddington J, Cooper CL, Field J, Goswami U, Huppert FA, Jenkins R, et al. The mental wealth of nations. Nature. 2008 Oct; 455(7216): 1057-60.

$46 \mathrm{Kim} \mathrm{H}$, Thyer BA, Munn JC. The relationship between perceived ageism and depressive symptoms in later life: understanding the mediating effects of self-perception of aging and purpose in life, using structural equation modeling. Educ Gerontol. 2019;45(2):10519.

47 Infurna FJ, Grimm KJ. The Use of Growth Mixture Modeling for Studying Resilience to Major Life Stressors in Adulthood and Old Age: Lessons for Class Size and Identification and Model Selection. J Gerontol B Psychol Sci Soc Sci. 2017 Dec;73(1):148-159.

48 Bonanno GA, Romero SA, Klein SI. The Temporal Elements of Psychological Resilience: An Integrative Framework for the Study of Individuals, Families, and Communities. Psychol Inq. 2015;26(2):139-69.

49 Ott CH, Lueger RJ, Kelber ST, Prigerson HG. Spousal bereavement in older adults: common, resilient, and chronic grief with defining characteristics. J Nerv Ment Dis. 2007 Apr; 195(4):332-41. 